Manuelle Medizin 2011 · 49:69

DOI 10.1007/s00337-011-0827-x

๑) Springer-Verlag 2011

\author{
L. Beyer \\ Ärztehaus Mitte, Jena
}

\section{Unsere Zeitschrift: Forum für wissenschaftliche Publikationen und Kommunikation praktischer Erfahrungen}

nen Gegenstand werden bis zu einem gewissen Grad durch neu hinzukommende neue Zeitschriften auf gesplittet.

Für die Mehrheit unserer Leser ist es sicherlich unerheblich, ob unsere Zeitschrift einen „impact factor" hat oder nicht; für den jungen Wissenschaftler ist es das leider nicht, denn seine Leistung wird danach bewertet. Allerdings lockt der breite Leserkreis, den unserer Zeitschrift zu bieten hat, doch auch zur Publikation von Originalarbeiten.

Für den Nachweis der komplexen Wirkung manueller Therapie sprechen die Ergebnisse einer Moskauer Forschergruppe zum Thema „Degenerativ-dystrophische Prozesse an der Halswirbelsäule und hämodynamisch bedingte vertebrobasiläre Insuffizienz“. Auf spezielle, doch alltägliche Faktoren, die aus Gewohnheit zu Dysfunktionen führen können, verweist der Beitrag zur „Untersuchung der Fußdruckverteilung hinsichtlich verschiedener Funktionsprinzipien des zahnärztlichen Fußanlassers“.

Die Verzahnung theoretischer Vorstellungen und praktischer Erfahrungen wird im Beitrag „Rückenschmerz - Schmerzverarbeitung und aktive segmentale Stabilisation“ deutlich. Ein etwas mehr wissenschaftstheoretischer Beitrag für alle, die doch einmal Daten aus ihren eigenen Untersuchungen sammeln wollen, geht der Frage nach „Was sind eigentlich Referenzdaten? Überlegungen zur Beurteilung von biomechanischer Messung und Referenzdatenkonzepten bei Patienten mit chronischen Rückenbeschwerden“. Für viele Leser von Interesse dürften auch die Argumente aus einem Urteil sein, dass ein Kollege für uns erstritten hat: „Abrechenbarkeit über Analogziffern und Erstattungsfähigkeit ärztlicher osteopathischer Leistungen“.

Etwas schwierig ist manchmal die Beurteilung der Veröffentlichung von Erfahrungsmeinungen aus der Praxis. Hier legen die Gutachter Wert auf exakte Zahlen der Untersuchungen, die exakte Zuordnung (Beschreibung) zu Patientengruppen, Messungen unter Standardbedingungen und Vergleich mit alternativer Behandlung.

Auch die Aufstellung von Hypothesen zu Indikationsstellungen und Therapieeffekten aus eigenen Erfahrungen und Kenntnissen ist wünschenswert. Wenn dazu noch keine Belege aus entsprechenden Studien vorliegen, sollten diese jedoch zur Überprüfung der Hypothese vorgeschlagen werden (nach Gutachten zu eingereichten Publikationen). Nur so lässt sich ein eventueller Vorwurf, mit Behauptungen zu argumentieren, vermeiden, und ein Wert für die Generierung neuer Studienfortbildung unserer Leser gewinnen.

Mit den besten Wünschen für Ihre tägliche Arbeit und entspanntes Lesen in einem schönen Frühling

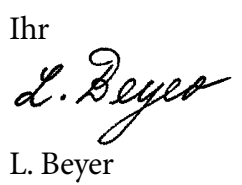

\section{Korrespondenzadresse \\ Prof. Dr. L. Beyer \\ Ärztehaus Mitte \\ Westbahnhofstraße 2, 07745 Jena \\ obeyer@t-online.de}

\section{Literatur}

1. Thesenpapier der Teilnehmer des 4. Berliner März 2011, www.bosch-stiftung.de Wissenschaftsgesprächs der Robert Bosch Stiftung und Transparenz der Datenerhebung gefordert. Auch Inhalte aus unserem eige- 